\title{
GESTIÓN DEL CAMBIO CLIMÁTICO DESDE LA UNIVERSIDAD
}

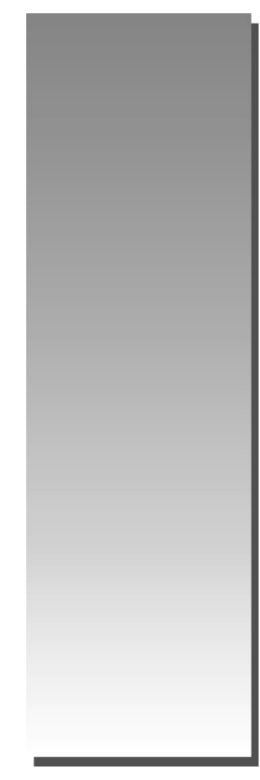

\author{
Yolanda Bustamante Sánchez* \\ yobusa22@yahoo.es
}

\section{RESUMEN}

Perú es considerado como el tercer país a nivel mundial más vulnerable al cambio climático por lo que la universidad, consciente de su papel motor de la sociedad, debe integrar el gobierno, empresa y sociedad, y gestionar para lograr su sostenibilidad en el corto, mediano y largo plazo, llenando los vacíos existentes y así alcanzar una mayor Responsabilidad Social Ambiental basada en el desarrollo de capacidades, la información, la comunicación, el uso de nuevas tecnologías mediante el trabajo en equipo, una visión compartida entre los diferentes actores sociales y financiamiento.

Palabras claves: universidad, cambio climático, fortalecimiento de capacidades, información, investigación, tecnología, financiamiento

\begin{abstract}
Peru is considered the third country in the world most vulnerable to climate change . The university, aware of its leading role in society, has to manage, integrate the government, business and society to achieve sustainability in the short, medium and long term, filling gaps achieving greater social responsibility based on environmental capacity building, information, communication, use of new technologies through teamwork, a shared vision between the different social actors
\end{abstract}

Keywords: university, climate change, capacity building, information, research, technology, financing.

\footnotetext{
* Licenciada en Ciencias de la Educación. Egresada de la Maestría en Administración con Mención en Recursos Humanos, Universidad Nacional Mayor San Marcos, Diploma en Gestión y Desarrollo Local, UIM, Granada, España. Estudios de especialización en Reducción de Riesgos de Desastres de la Organización Internacional del Trabajo, OIT, Turín, Italia.Docente Investigadora y publicaciones en Vicerrectorado de Investigación (UNMSM), ESAN, Red Nacional de Documentación e Información sobre Administración Pública/Instituto Nacional de administración Pública, INAP (Argentina), Centro Latinoamericano de Administración para el Desarrollo, CLAD (Venezuela), Ponencias Congresos Internacionales en Internet-Eumed.net
} 


\section{INTRODUCCIÓN}

Perú es considerado a nivel mundial como el tercer país más vulnerable al cambio climático, por lo que tenemos el compromiso de informar sobre su vulnerabilidad y presentar propuestas de mitigación y adaptación para disminuir impactos adversos producidos por la contaminación atmosférica.

Es a partir del cambio climático, proceso de largo plazo influido por actividades humanas que concentran gases de efecto invernadero en la atmósfera mundial y que tendrá impactos importantes en la economía, sociedad y capital natural, que se precisa estabilizar las concentraciones de gases de efecto invernadero en la atmósfera a un nivel que impida interferencias antrópicas peligrosas en el sistema climático asegurando que los ecosistemas se adapten naturalmente al cambio climático, permitiendo que el desarrollo económico prosiga de manera sostenible.

En este contexto, la universidad, consciente de su papel motor de la sociedad, debe vertebrar el crecimiento de sus infraestructuras e instalaciones para contribuir al impulso del concepto de desarrollo sostenible, debe concientizar desde su eje estratégico para conseguir mayores niveles de bienestar y de riqueza.

Crecimiento y sostenibilidad basados en el trabajo integrado de universidad, gobierno, empresa y sociedad para equilibrar las políticas de desarrollo y que sean eficientes en el corto, mediano y largo plazo, llenando los vacíos existentes para lograr una mayor responsabilidad social ambiental, mejorando la conciencia ambiental basada en la informacion, comunicación, uso de nuevas tecnologías mediante el trabajo en equipo y una visión compartida, desarrollando capacidades y promoviendo el desarrollo local sostenible.

\section{MARCO CONCEPTUAL}

\section{CAMBIO CLIMÁTICO}

El Ministerio del Ambiente, autoridad ambiental nacional en el Perú creada por Decreto Legislativo 103 de mayo de 2008, cuenta con una Dirección General de Cambio Climático, Desertificación y Recursos Hídricos (DGCCDRH).

A partir del 1992, el gobierno focalizó sus esfuerzos en ejecutar las medidas destinadas a mitigar las emisiones de gases de efecto invernadero, a reducir la vulnerabilidad y mejorar la capacidad de adaptación de la población al cambio climático
Según los objetivos de desarrollo del milenio, el Perú culminó su suscripción en el Acuerdo Nacional. La Estrategia Nacional de Cambio Climático ENCC, aprobada en 2003, consta de once líneas estratégicas de acción para la reducción de los impactos adversos al cambio climático a través de estudios integrados de vulnerabilidad y adaptación y el control de emisiones de contaminantes locales de gases de efecto invernadero (GEI) a través de energías renovables y de eficiencia energética en los diversos sectores productivos.

Hay otro documento importante que provee el marco para la elaboración de políticas sobre cambio climático: la estrategtia nacional de competitivad que propone el uso racional de los recursos naturales y el medio ambiente, genera investigación, propone el uso de tecnologías limpias para abrir nuevos mercados a las exportaciones nacionales.

\section{EFECTO INVERNADERO}

El efecto invernadero es un fenómeno natural necesario para la vida en la Tierra. Sin este, la temperatura sería de 18 grados bajo cero y no tendríamos agua en forma líquida. El efecto invernadero es la absorción de parte de la radiación solar que es reflejada por la Tierra, ello hace que la temperatura promedio del planeta sea aproximadamente 15 grados centígrados. Esta absorción se produce por los gases de efecto invernadero (GEI) especialmente el dióxido de carbono, metano y óxido nitroso. Sin embargo en los últimos 200 años, en la era industrial, las actividades humanas han aumentado su concentración en la atmósfera a niveles mayores. Además el hombre ha fabricado gases no naturales: gases fluorados que dañan el ozono. La quema de combustibles fósiles, la deforestación y la urbanización han incrementado la concentración de los gases de efecto invernadero incidiendo en el cambio climático.

Solo un $40 \%$ del $\mathrm{CO}_{2}$ extra que emitimos a la atmósfera es absorbido por los organismos que realizan fotosíntesis, principalmente los del mar. El resto pasa a aumentar el nivel del $\mathrm{CO}_{2}$ en la atmósfera.

Desde hace 50 años, las emisiones de GEI están aumentando en el planeta originando el calentamiento global, lo que se evidencia a través de la desglaciación o pérdida de los glaciares. El país, depende en su gran mayoría de los nevados para el suministro de agua potable, generación de energía y riego de cultivos. 
El inventario de gases de efecto invernadero que emite el país se refiere a las emisiones de estos gases y su evolución a fin de dimensionar, orientar y promover los esfuerzos nacionales de mitigación de emisiones y contribuir a la meta global de lucha contra el cambio climático. A nivel nacional, el inventario de GEI permite orientar la normatividad para los sectores económicos y sociales, incentivar las alternativas eficientes de bajo costo para el uso de energía y de los recursos naturales y establecer las políticas que aseguren un desarrollo sostenible mejor adaptado a las exigencias del cambio climático.

La Tierra, como casi todo objeto en el universo, emite energía por radiación a una razón que aumenta con la temperatura. La temperatura en la Tierra tiene un promedio de 15 grados Celsius, lo cual corresponde a que esta radiación sea principalmente en la banda infrarroja.

La pérdida continua de energía llevaría a un enfriamiento del planeta pero la radiación recibida del sol compensa esta pérdida, de manera que se logra un balance energético.

Para la preparación del inventario se ha considerado las directrices técnicas y formales de las Comunicaciones Nacionales de las Partes de la Comisión Marco de las Naciones Unidas del Cambio Climático (CMNUCC), así como las directrices del Panel Intergubernamental sobre Cambio Climático (IPCC).

Las principales fuentes de emisiones nacionales se clasifican por categoría: energía, procesos industriales, agricultura, uso del suelo, cambio de uso de suelo y silvicultura (USCUSS) y desechos.

\section{AVANCES EN MITIGACIÓN DEL CAMBIO CLIMÁTICO EN EL PERÚ}

Se está llevando a cabo medidas conducentes a optimizar el uso de combustibles fósiles y hacer más eficiente la matriz energética, pero sobre todo a reducir la mayor fuente de emisiones: la deforestación y el cambio de uso del suelo en bosques amazónicos. Los esfuerzos han de orientarse al incremento de la eficiencia energética, priorizar la generación hidroeléctrica y desarrollar el potencial de energías renovables y limpias.

En el campo forestal se presentan los avances en la institucionalidad y el marco de políticas sobre acciones de forestación y reforestación, incluyendo el Programa Nacional de Conservación de Bosques para la mitigación del cambio climático en cuyo marco se implementará el mecanismo REDD+.

En el sector energético se presentan progresos en la promoción de la energía renovable (hidráulica, eólica y solar) los biocombustibles y el uso del gas natural.

De acuerdo a la clasificación del IPCC, las emisiones del sector energético están constituidas por aquellas provenientes del consumo de combustibles en todos los sectores productivos del país, la generación de electricidad, las industrias, el transporte, la agricultura, el sector residencial y comercial. El Estado ha fomentado la producción y el uso de energías renovables a través de la aprobación de diversas normas legales que promueven la inversión en la generación eléctrica basada en energías renovables. En el Decreto Legislativo 1002 del año 2008, la Promoción de la Inversión para la Generación de Electricidad con el uso de Energías Renovables considera como (RER) a biomasa, energía eólica, solar, geotérmica, mareomotriz e hidroeléctrica. Está previsto que el Minem elabore el Plan Nacional de Energías Renovables que se enmarque en un Plan Nacional de Energía, el que incluirá estrategias, programas y proyectos de energía renovable que mejoren la calidad de vida de la población y el ambiente.

En el 2000 se aprobó la ley sobre la Política de Eficiencia Energética N. ${ }^{\circ} 27345$ que declara de interés nacional la promoción del uso eficiente de la energía (UEE) para asegurar el suministro de energía, proteger al consumidor.

\section{ENERGÍA HIDROELÉCTRICA}

El potencial hidroeléctrico de el país fue evaluado en 1979 con apoyo de la Cooperación Técnica Alemana (GTZ) estimándose un potencial técnico de 58,937 MW (Minam 2009). Sin embargo, el desarrollo de la energía hidroeléctrica ha sufrido una política de desincentivación entre setiembre de 1998 y marzo de 2001 en que se suspendió la concesión temporal o definitiva de generación hidráulica (Leyes 26980 y 27133) a fin de promover la industria del gas natural, lo que evitó el ingreso de nuevos concesionarios de generación hidroeléctrica.

\section{ENERGÍA EÓLICA}

En 2007, la generación eléctrica con fuentes renovables no convencionales en Perú (especifica- 
mente con viento) fue del menos del $1 \%$. A fin de promover la inversión en este tipo de energía, se actualizó el mapa eólico del país, identificándose una capacidad de 2000 MW y determinándose las zonas de mayor potencial para el desarrollo de parques eólicos (Minam 2009). Hasta inicios del 2009, el Minem había entregado más de 60 concesiones temporales para la ejecución de estudios del desarrollo de centrales eólicas.

\section{ENERGÍA SOLAR}

El Atlas de Energía Solar del Perú del año 2003 demuestra que tenemos un potencial promedio de $5.24 \mathrm{Kwh} / \mathrm{m}^{2}$. Según el Minem, el Perú cuenta con una potencia instalada de $3.73 \mathrm{Mwp}$. Las potencialidades y barreras para la generación de energía solar fotovoltaica figuran que Perú es un país con altos niveles de radiación solar en la sierra y algunos departamentos de la costa. Existen algunas tecnologías maduras que se emplean para el calentamiento de agua (termas solares). Se han desarrollado muchos proyectos en el país que emplean sistemas fotovoltaicos como fuente de energía. Hay organismos de cooperación internacional que promueven el uso de la energía solar fotovoltaica.

Sin embargo, hay muchos proyectos que se han implementado sin tener en cuenta la sostenibilidad en el tiempo de la instalación, hay un alto nivel de informalidad en las zonas rurales. Existe un mercado negro con componentes de baja calidad que son comercializados y hay robo de sistemas instalados para luego comercializarse en dicho mercado negro.

\section{BIOCOMBUSTIBLES}

En la última década han sido importantes los esfuerzos normativos y la inversión privada para promocionar la producción y comercialización de biocombustibles en el país. La ley 28054 establece el marco legal para promover el desarrollo de biocombustibles, la inversión en la producción y comercialización de estos. Entidades como el Minem apoyan en esta labor, el programa de uso de biocombustibles (PROBIOCOM) a cargo de Proinversión constituye un ejemplo. Se promueve el uso de gas natural.

\section{HACIA UN PLAN NACIONAL DE MITIGACIÓN}

El MINAM cuenta con una Plan Nacional de Mitigación cuyo objetivo es implementar políticas y medidas de mitigación de las causas del cambio climático en el Perú en cinco sectores: energía, transporte, industria, manejo de desechos, forestal y uso de suelos.

En el sector energético: las Medidas Nacionales Apropiadas de Mitigación (NAMA, por sus siglas en inglés), en coordinación con el Ministerio de Energía y Minas y el Ministerio del Ambiente (Minam) vienen diversificando las energías renovables, la eficiencia energética, las industrias, servicios residenciales calidad de los combustibles, cambio en las normas de emisiones de fuentes fijas, calidad de los combustibles importados y de las refinerías. Asimismo, se promuevan nuevos proyectos mineros que cuenten con generación de energías renovables, se implementan programas de ahorro y uso eficiente de energía en el sector público.

En el sector transporte, las NAMA, en coordinación con el Ministerio de Transporte y Comunicaciones y el Minam controlan las emisiones en el sector terrestre proveniente del parque automotor, promoviendo el uso del gas natural.

En el sector industria, el grupo Nama coordina con el Ministerio de la Producción y el Minam la actividad de las industrias manufactureras, como las sementeras, siderúrgicas y ladrilleras para reducir las emisiones por combustión, velando por el cumplimiento de la normativa ambiental vinculan a los GEI.

En el sector agrícola el grupo NAMA coordina con el Ministerio de Agricultura (Minag) el manejo del ganado, los residuos agropecuarios, los sistemas agrícolas y manejos de suelo.

El NAMA en el sector forestal y uso de suelo brinda aporte significativo para el Plan Nacional de Mitigación, por ser la principal causa de la deforestación (agricultura migratoria, ganadería, apertura de trochas, carreteras o vías de penetración y minería informal. Coordinan con el Minam y el Minag la conservación de los bosques en las Áreas Naturales Protegidas, el manejo integrado de suelos, las políticas de desarrollo social, los Sistemas de Información y control forestal, los proyectos MDL de reforestación y el mecanismo emergente REDD+.

En lo que refiere a los desechos, el grupo NAMA orienta el manejo adecuado de los desechos causantes del $90 \%$ de emisiones de metano proveniente de los residuos sólidos depositados en rellenos sanitarios o botaderos, $60 \%$ de los 
cuales son generados en Lima. De esta manera se impulsa el reciclaje, compostaje, implementación de programas de manejo y tratamiento de aguas residuales, y uso de residuos agropecuarios: biomasa para la producción energética con biodigestores, calderos.

\section{AVANCES EN ADAPTACIÓN AL CAMBIO CLIMÁTICO Y EL ESTADO DE LA VULNERABILIDAD EN EL PERÚ}

La adaptación al cambio climático consiste en el ajuste en los sistemas naturales o humanos a los estímulos climáticos reales o esperados o a sus efectos.

Las amenazas crecientes son las sequías, fuertes lluvias, inundaciones, heladas, granizadas cuyo registro denota un crecimiento de más de seis veces de 1997 a 2006, generando la intensificación del Fenómeno de El Niño (FEN). Los estudios demuestran que el régimen de temperaturas y precipitaciones están cambiando a lo largo del país y que se incrementarán en el tiempo. Para el 2030, la temperatura mínima del aire aumentaría entre 0.4 y 1.4 en la costa y selva norte, sector central y parte del sector sur andino. Asimismo las precipitaciones en el 2030 muestran deficiencias en la sierra, la selva norte y central, con incrementos en la costa norte y selva sur.

Los estudios realizados en el norte del Perú estiman un incremento del nivel del mar entre 60 y 81 centímetros para los próximos cien años.

En los últimos 30 años se perdió el $22 \%$ de la superficie glaciar generando una pérdida de 12,000 millones de metros cúbicos de agua. Para el 2030 la disponibilidad hídrica en la vertiente del Pacífico disminuiría en 6\% . La deglaciación no solo tiene un impacto en la disponibilidad de agua, sino que aumenta el riesgo de aludes y aluviones por incrementarse el número de lagunas colgantes.

\section{MARCO NORMATIVO DEL CAMBIO CLIMÁTICO}

1993: Constitución Política del Perú que establece el derecho constitucional a gozar de un ambiente equilibrado y adecuado para el desarrollo de la vida.

1993: Creación de la Comisión Nacional de Cambio Climático (MINAM/CONAM) por Resolución Suprema 359-RE.

1996: Se presenta y reporta la Agenda Ambiental Nacional como principal instrumento de planificación y gestión ambiental nacional, sectorial y local.
2000: Norma para la aplicación del Protocolo de Montreal relativo a las sustancias que agotan la capa de ozono.

2001: Reglamento de estándares nacionales de calidad ambiental del aire.

2001: Creación de la Comisión Nacional para el Ordenamiento Territorial Ambiental y la Zonificación Ecológica Económica (ZEE) en todo el país.

2002: Ley Orgánica de Gobiernos Regionales, Ley 27867 que establece la obligación de generar Estrategias Regionales de Cambio Climático y Diversidad Biológica.

2004: Ley del Sistema de Gestión Ambiental promulgada mediante Ley 28245 que establece que CONAM (hoy ministerio de ambiente) sea la institución encargada del diseño y dirección participativa para las obligaciones derivadas de la Convención Marco de las Naciones Unidas sobre Cambio Climático (CMNUCC), coordinar la elaboración periódica de los informes nacionales, comunicación nacional.

2005: Se prioriza la implementación de la Estrategia Nacional de Cambio Climático con el enfoque de incorporar la variable climática en los planes de desarrollo a través de la Agenda Nacional Ambiental.

2005: Ley general del ambiente, Ley 28611 que establece la implementación de un Sistema Nacional de Gestión Ambiental en forma conjunta con la comisiones ambientales regionales y la autoridad ambiental nacional, el Minam, promueve bonos de descontaminación y mecanismos alternativos para acceder a fondos creados al amparo del Protocolo de Kyoto.

2007: Ley de Eficiencia Energética, aprobada por Decreto Supremo 053-2007-MINEM del 2000, para el uso eficiente de la energía, proteger al consumidor, fomentar la competitividad de la economía nacional, reducir el impacto ambiental negativo del uso y consumo de energía.

2008: Creación del Ministerio del Ambiente.

2008: Creación de la Autoridad Nacional del Agua, ANA y del Sistema Nacional de Recursos Hídricos iniciativa para avanzar en la gestión del agua a nivel nacional.

2008: El Ministerio de Agricultura MINAG, crea el Grupo de Trabajo Técnico de Seguridad Alimentaria y Cambio Climático (GTTSACC), mediante 
Resolución Ministerial 0647-2008-AG, a fin de proponer la visión sectorial del cambio climático en los sistemas productivos agrarios del país y recomendar medidas que orienten procesos y acciones institucionales, así como la articulación intersectorial para la adaptación al cambio climático mediante la reducción de la vulnerabilidad del agro, teniendo como finalidad la contribución a la seguridad agroalimentaria del Perú.

En los períodos legislativos 2006-2008 entró en funcionamiento la Comision Especial Muiltipartidaria denominada Cambio Climatico y Biodiversidad del Congreso de la República encargada de evaluar y proponer lineamientos y políticas destinadas a proteger el medio ambiente y la biodiversidad del país.

Mediante Decreto Supremo 075-2009-PCM se creó la Comisión Multisectorial sobre Bioenergía, conformada por el Ministerio Energía, Agricultura, Desarrollo estratégico de los Recursos Naturales y de Mype e industria para evaluar y recomendar medidas que promuevan la gestión integral de la bioenergía en el país. Esta comisión está adscrita al Ministerio de Agricultura.

2009: Política Nacional del Ambiente que constituye el conjunto de lineamientos, objetivos, estrategias, metas, programas e instrumentos de carácter público que tiene como propósito definir y orientar las acciones de las entidades del gobierno nacional, regional y local del sector privado y sociedad civil en materia ambiental. La Política Nacional del Ambiente y sus cuatro ejes de política son la base para la formulación del Plan Nacional de Acción Ambiental y ha sido elaborada tomando en cuenta la Declaracion de Río y los Objetivos de Desarrollo del Milenio y constituye uno de los principales instrumentos de gestión para el logro del desarrollo sostenible en el país. De este modo el Minam ha iniciado el proceso de la formulación del Plan Nacional de Acción Ambiental (2010-2021) -PLANAA, teniendo como marco la Política Nacional del Ambiente.

2009: Creación del Centro Nacional de Planeamiento Estratégico (Ceplan) y El Sistema Nacional de Planeamiento Estratégico (Sinaplan). El Minam ha fortalecido la institucionalidad ambiental pues ha permitido la fusión del Conam y la adscripción del SENAMHI, del Instituto Geofísico del Perú (IGP), del Organismo de Evaluación y Fiscalización Ambiental (OEFA), del Instituto de Investigaciones de la Amazonía Peruana (IIAP) y parte del Instituto Nacional de Recursos Naturales (Inrena) con la cual formó el Servicio Nacional de Áreas Naturales Protegidas (SERNANP). Se ha creado el Viceministerio de Gestión Ambiental y del Desarrollo Estratégico de los Recursos Naturales (ENCC), asignándosele a este último la coordinación y supervisión de ENCC y de Lucha contra la Desertificación y la Sequía (PNUD-MINAM, 2009). La sociedad civil, empresas, universidades y otros estamentos vinculados al desarrollo tienen un creciente interés en el análisis de la problemática del cambio climático en el Perú, con participación en acciones concretas para la reducción de emisiones y gestión de riesgos así como la formación de capacidades, generación de conocimientos y aportes metodológicos para la toma de decisiones.

\section{MÉTODOS}

Gestionar el cambio climático desde la universidad es un asunto de inminente prioridad para el país en su camino al desarrollo sostenible, por lo que se precisa considerar cinco pilares de acción: fortalecimiento de capacidades, información, investigación y observación sistemática, tecnología y financiamiento.

\section{FORTALECIMIENTO DE CAPACIDADES, EXU- DACIÓN Y SENSIBILIZACIÓN}

En las instituciones del Estado y sus diferentes niveles para una buena toma de decisiones, establecimiento de plataformas de coordinación efectivas a nivel nacional, sectorial, regional y local, desarrollando una cultura de prevención, gestión de conflictos y toma de decisiones en un contexto de alta incertidumbre y escasez de recursos, dándose prioridad a la planificación a largo plazo con acciones en el corto plazo que reduzcan la vulnerabilidad.

\section{INFORMACIÓN}

Se requiere generar información de fácil acceso, sistematizada en base a estadísticas del clima, hidrológicas y meteorológicas, censos y estadísticas ambientales sobre el estado de los ecosistemas, a la investigación de los impactos del cambio climático en los distintos sectores, ecosistemas y al uso y acceso de esta información por distintos usuarios. Las acciones de comunicación y difusión de información convocarán la participación del MINAM, MINISTERIO DE EDUCACIÓN, UNIVERSIDADES y organizaciones de sociedad civil. 


\section{INVESTIGACIÓN Y OBSERVACIÓN SISTEMÁTICA}

La investigación tomará en cuenta el cambio climático, sus impactos según las metas del Plan Estratégico de Desarrollo Nacional 2021, el Marco Macroeconómico Multianual, el Plan Nacional de Competitividad y los programas de reducción de pobreza. Asimismo, los Planes Estratégicos, Planes de Ordenamiento Territorial y la Zonificación Ecológica Económica, instrumentos regionales como los Planes de Desarrollo Concertados y los instrumentos presupuestales.

\section{TECNOLOGÍA}

Las medidas de adaptación ante distintas amenazas del cambio climático (lluvias extremas, escases de agua, variación en la temperatura y precipitación requieren de la aplicación de tecnologías tanto para la gestión del conocimiento como para la infraestructura.

Las tecnologías para la adaptación puede servir para evitar, controlar, revertir los impactos del cambio climático, recuperar las tecnologías campesinas basada en saberes ancestrales andinos que han servido para la adaptación autóctona ante el cambio climático y que representan un reto para su recuperación, valoración y aprovechamiento

\section{FINANCIAMIENTO}

El Perú requiere introducir nuevas líneas e instrumentos innovadores de financiamiento para la adaptación, ajustando mecanismos, instrumentos y plataformas existentes que permitan conjugar diversas fuentes: la cooperación y financiamiento internacional, el sector privado y el presupuesto del Estado en sus diferentes niveles.

EL Financiamiento para la adaptación al cambio climático debe ser integrado dentro de las políticas y programas de desarrollo del gobierno como una inversión. Debe explorarse mecanismos para involucrar al sector privado en la adaptación ya que maneja la mayor cantidad de recursos financieros

\section{RESULTADOS}

El cambio climático es definitivamente uno de los temas más relevantes en la agenda ambiental internacional de la economía, el comercio y las decisiones políticas en el actual mundo globalizado. La aplicación de la metodología arriba propuesta conllevará al logro siguiente:
1. Desarrollo de capacidades en cambio climático potenciando los sistemas tradicionales de conocimiento y tecnológicos para el sector público: población y empresas.

2. Procesos de desarrollo a través de una planificación concertada, generando la reducción de la vulnerabilidad.

3. Ordenamiento Territorial mediante Planes de Zonificación Ecológica y Económica

4. Participación activa y permanente en el ámbito nacional, regional local con elementos críticos, constructivos, proactivos.

5. Visión compartida que aborda adecuadamente los problemas existentes.

6. Proceso de toma de decisiones con políticas preventivas para la protección ambiental, social y la economía.

7. Desarrollo de capacidades basados en los conocimientos actuales sobre las consecuencias sociales que podrían tener estos fenómenos son bastante escasos.

8. Información actualizada, uniforme y adecuada.

9. Investigación y valoraciones objetivas esenciales para comprender mejor los problemas ambientales, proyectos e ideas ambientales plasmados en un planteamiento integrador y coordinado.

10. Tecnologías limpias que cuidan el medio ambiente, permitiendo la mitigación y adaptación al cambio climático.

11. Un financiamiento acertado permite la cooperación y apoyo internacionales del sector público y privado.

\section{CONCLUSIONES}

1. Un personal apto, debidamente capacitado puede ejercer labores de supervisión, fiscalización permitiendo un trato responsable de labor asignada.

2. Las competencias compartidas por el gobierno nacional, regionales o locales definen un mecanismo de recopilación de información, delimitando las funciones y competencias de cada sector en el marco del cambio climático.

3. Una tecnología actualizada permitirá fortalecer capacidades en los diversos sectores de actuación. 
4. Un plan de acción organizado permitirá recolectar datos, niveles de actividad, elaboración de informes respectivos. Información pertinente al Sistenma Nacional de Inventario de Gases de Efecto Invernadero (SNINGEI), se constituirá en una base de datos actualizada, confiable para ser utilizada y aplicada según los requerimientos.

5. La universidad, como ente gestor e integrador de los actores sociales, permitirá el logro de una Plan de Acción Nacional para enfrentar el cambio climático.

\section{BIBLIOGRAFÍA}

CALVO BUENDÍA, Eduardo (2010). Guía Metodológica para la adaptación a los impactos del cambio climático en las ciudades y opciones de Mitigación de emisiones de Gases de Efecto Invernadero, Foro ciudades para la vida. Lima: www.ciudad.org.pe

CAMBIO CLIMÁTICO Y DESPLAZAMIENTO. N. ${ }^{\circ} 31$ -Noviembre 2008 Migraciones ForzadasRevista Centro De Estudios Sobre Refugiados. Imprenta Universidad Alicante.

www.migracionesforzadas.org.

COSA SERIA ESTE CLIMA- Panorama del cambio climático en la Comunidad Andina.

Publicado por la Secretaría General de la Comunidad Andina, el Programa de las Naciones Unidas para el Medio Ambiente, Oficina Regional para América Latina y el Caribe y la Agencia Española de Cooperación Internacional.

www.comunidadandina.org

COMUNICACIÓN NACIONAL DEL PERÚ (2001) a la Convención de Naciones Unidas sobre Cambio Climático-Primera Comunicación. CONSEJO NACIONAL DEL AMBIENTE - JUNIO 2001.

DIAGNÓSTICO DE LA SITUACIÓN DEL MANEJO DE RESIDUOS SÓLIDOS MUNICIPALES EN AMÉRICA LATINA Y EL CARIBE.

Guido acurio, antonio rossin, paulo fernando teixeira, francisco zepeda.
$2^{A}$ edición: set (1998) - serie ambiental 18organización panamericana de la salud/oms. http://www.cepis.org.pe

EDUCAR PARA EL AMBIENTE. La construcción de conocimiento como espacio participativo. Metodología y guías didácticas (2003). Buenos Aires: Publicación Financiada con Fondos de La Coperación Técnica de La República Federal de Alemania. Isbn: 98720906-4-5

EL PERÚ Y EL CAMBIO CLIMÁTICO- Segunda Comunciación Nacional del Perú a la Convención Marco de Las Naciones Unidas sobre Cambio Climático (2010).

Impresiones \& Ediciones Aguilar S.A.C.

Diseño Gráfico Diagramación e Infografías: Libelula Comunicación, Ambiente y Desarrollo S.A.C.

Depósito Legal en La Biblioteca Nacional del Perú Nro 2010-07724

GUÍA PARA LA ELABORACIÓN DE LA ESTRATEGIA REGIONAL FRENTE AL CAMBIO CLIMÁTICO. Ministerio Del Ambiente. Segunda Comunicación Nacional de Cambio Climático

Depósito Legal en La Biblioteca Nacinal del Perú N. ${ }^{\circ}$ 2009-05214Bn

LECCIONES APRENDIDAS EN LA GESTIÓN DEL RIESGO EN PROCESOS DE PLANIFICACIÓN E INVERSIÓN PARA EL DESARROLLO (2010).

Memoria del Taller Internacional. Perú, 19 al 22 Julio 2010.

Instituciones Organizadoras: Ministerio Economía y Finanzas Perú, Gobierno Regional Piura, Giz, Cosude, Maec.

Perú, Marzo De 2011.

PLAN NACIONAL DE ACCIÓN AMBIENTAL - PLANAA - PERÚ 2011-2021 - 2. ${ }^{\text {a }}$ ed. APROBADO POR D.S. 014-2011- MINAM (2011). Publicado por diario El Peruano , 09 Julio 2011.

RUSSI DANIELLA, Puig Ventosa, Ignasi, Ramos Martín, Jesús, Ortega Cierda, Miguel, Ungar, Paula. (2003). Deuda ecológica- ¿Quien debe a quién?. Colectivo de difusión de la deuda ecológica, CDs. Icaria Editorial 
Cátedra Unesco para la sosteniblidad de la universidad politécnica de catalunya. www. observatoriodeuda.org

SÁENZ ORLANDO. Experiencia latinoamericana en la incorporación de la dimensión ambiental en la universidad.

ZEGARRA PERALTA, Augusto - PCM. Programa del Prev y La visión global de la Ley 29664- Ley del Sinagerd (Sistema Nacional De Gestión Del Riesgo)
ZONIFICACIÓN ECOLÓGICA, ECONÓMICA Y GOBIERNOS LOCALES - Guía Metodológica (2007). Consejo Nacional del AmbienteCONAM. http://www.conam.gob.pe 